Case Report

\title{
Clinical and imaging features in a patient with hemifacial hyperplasia
}

\author{
Roseli T. Miranda ${ }^{1)}$, Letízia M. Barros ${ }^{1)}$, Luis A. Nogueira dos Santos ${ }^{2,3)}$, \\ Paulo R. F. Bonan ${ }^{3)}$ and Hercílio Martelli Jr ${ }^{1,3)}$ \\ ${ }^{1)}$ Department of Oral Diagnosis, Dental School, University of Alfenas, Alfenas, MG, Brazil \\ ${ }^{2}$ Faculty of Dentistry Piracicaba, State University of Campinas (UNICAMP), \\ Piracicaba, SP, Brazil \\ ${ }^{3)}$ Stomatology Clinic, Dental School, State University of Montes Claros, Montes Claros, MG, Brazil
}

(Received 10 August 2009 and accepted 10 June 2010)

\begin{abstract}
Hemifacial hyperplasia is a developmental disorder characterized by marked unilateral facial asymmetry. It involves the hard and soft tissues of the face. The cause is unknown, although several predisposing factors have been described. A case report of an 8-year-old boy with hemifacial hyperplasia is presented to highlight the clinical and imaging findings. (J Oral Sci 52, 509-512, 2010)
\end{abstract}

Keywords: hemifacial hyperplasia; developmental anomaly; facial asymmetry.

\section{Introduction}

Hemifacial hyperplasia (HFH), described initially by Merckel in 1822 and Wagner in 1839 , is a sporadic congenital condition. HFH is a segmental form of congenital hemihyperplasia (1); the classic presentation is a unilateral overgrowth of the orofacial soft tissues, bones, and teeth (2). The right side of the face is affected more often than the left side. HFH is more common in men than in women and in white people than in other racial groups (3). It may be associated with other conditions, such as acromegaly and pituitary gigantism, or with hypertrophy of other parts of the body (4).

Correspondence to Dr. Luis Antônio Nogueira dos Santos, Rua Irma Beata, 380 Centro CEP 39400-110, Montes Claros, MG, Brazil

Tel: +55-38-3201-8041

Fax: +55-38-3221-3517

E-mail: luisnogueira@ radiocenterdigital.com
Many theories have been proposed regarding the cause of $\mathrm{HFH}$, including hormonal imbalances; diseases involving the neural system; vascular conditions such as hemangiomas and arteriovenous malformations; abnormalities such as lymphangioma, incomplete twinning, abnormal intrauterine environment, somatic mutations, and central nervous system lesions; mechanical influences; and congenital syphilis. Thus, many authors consider the development of prenatal teeth to be a good indication of future malformations (4). The aim of this study was to describe one sporadic case of HFH affecting an 8-year-old boy, and to discuss the clinical and imaging features of this condition.

\section{Case Report}

An 8-year-old white boy was referred to the Dental School of University of Alfenas, Minas Gerais State, Brazil, with a chief complaint of abnormal shape of the jaw and face on the right side. The boy's mother reported that his face and jaws were of normal size until the age of 2 years, when he was punched on the right side of his jaw. Subsequent to this, his face became deformed, with mainly his jaw developing. He was born to non-consanguineous parents at full term by cesarean section. There were no complications during pregnancy and no noteworthy postnatal illness was reported. His parents and three brothers were clinically normal and no family history of abnormalities of facial or gnathic anatomy was reported. Extra-oral examination revealed enlargement of the right side of the face, extending to the submandibular midline near the symphysis menti. The lips were asymmetric and appeared very dry (Fig. 1A). On palpation, soft tissue 


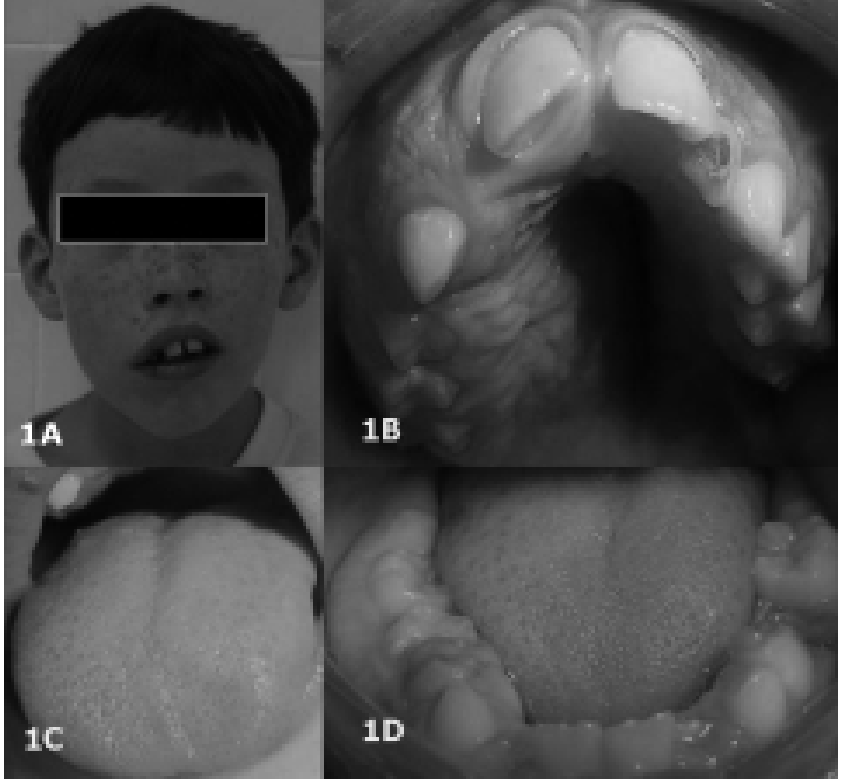

Fig. 1 (A) Abnormal shape of the jaw and face on the right side. Note the asymmetry of the lips, cheeks, and chin. (B) Clinical intra-oral picture showing the maxillary region. Gingival enlargement is more evident in the posterior region, where the gingiva partially cover the crowns of the teeth. A deep, arched palate was also found. (C) Clinical intra-oral picture showing growth discrepancy on the right side of the tongue. (D) Clinical intra-oral picture showing the mandibular region. The enlarged tissue appeared cyanotic, firm, dense and of fibrous consistency, causing difficulties in maintaining adequate oral hygiene.

enlargement and bone enlargement were noted. There was no submandibular lymphadenopathy and no physical abnormalities elsewhere in the body. Serum calcium, phosphate, and alkaline phosphatase levels were within normal limits.

Intra-oral examination revealed an arched palate with a prominent depression in the middle portion, lateral cross bite, anterior open bite, and malocclusion with overjet and overbite. Gingival enlargement was most evident in the posterior region, where the gingiva partially covered the tooth crowns. In the mandible, posterior enlargement was associated with increased soft tissue on the right side of the oral cavity. The tissue appeared cyanotic, firm, dense, and of fibrous consistency, causing difficulties in maintaining adequate oral hygiene. The growth discrepancy was also noted on the right side of the tongue (Figs. 1BD).

Panoramic radiography showed agenesis of the maxillary first premolar on both sides and asymmetric mandible (Fig. 2A). To better define the facial deformity, spiral
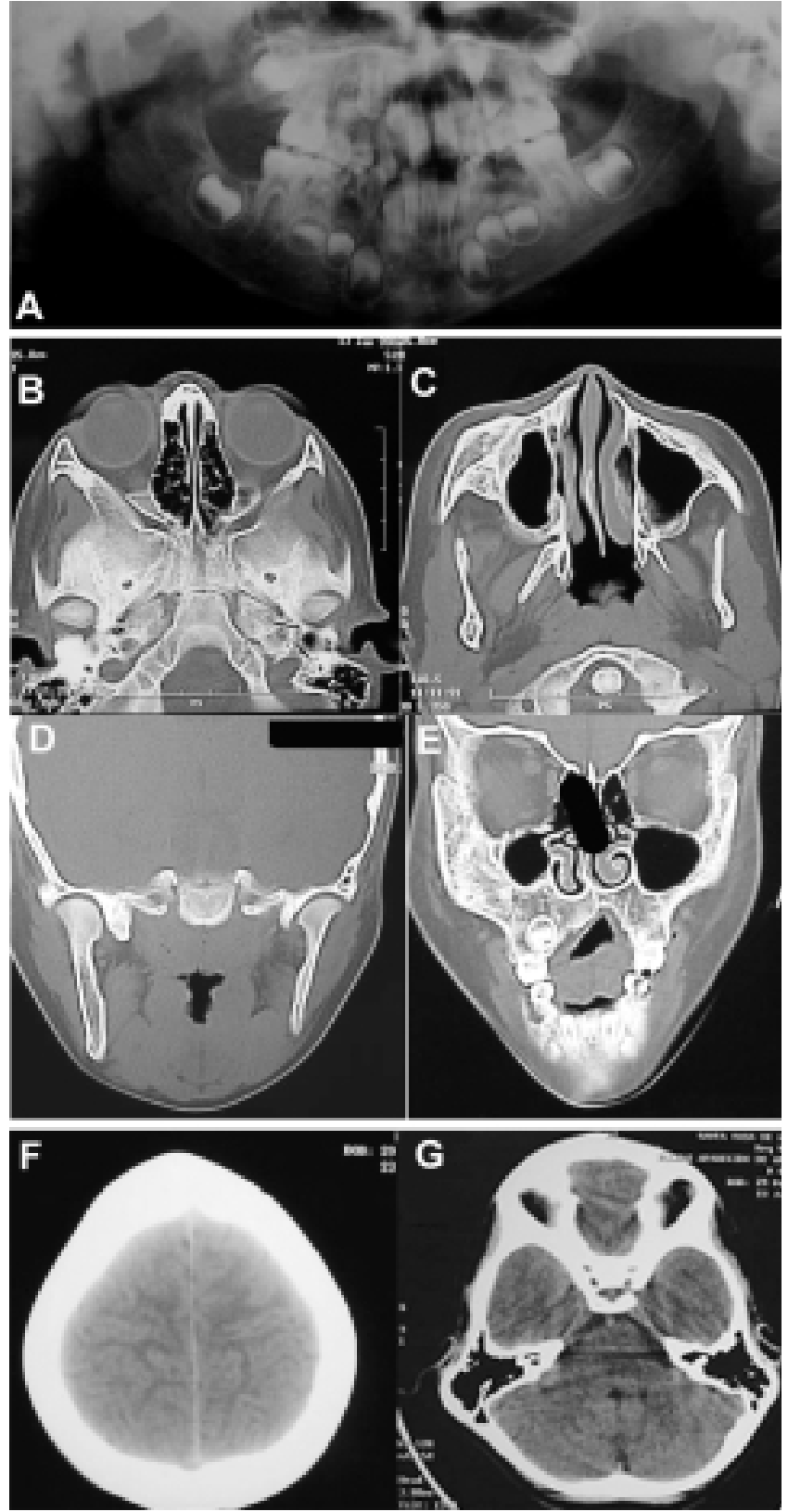

Fig. 2 (A) Panoramic radiograph showing agenesis of the maxillary first premolar on both sides and an asymmetric mandible. $(\mathrm{B}, \mathrm{C})$ Axial plane: spiral CT shows irregular and hyperdense growth of the right hemiface with involvement of the zygomatic and frontal bones, temporal and maxillary process, sphenoid, occipital bone, maxilla (enlargement of the cheek and palate), and mandibular ramus and condyle. (D,E). Coronal plane: hypoplasia of the right maxillary antrum associated with an increase in the vertical dimensions of the orbit. Enlargement of the inferior turbinate with nasal septum deviation is observed. (F,G) Brain CT shows no abnormalities other than enlargement of the frontal and parietal bones.

computed tomography (CT) of the face and skull was 
performed. The following parameters were used: slice thickness of $3.0 \mathrm{~mm}$ (skull) and $5.0 \mathrm{~mm}$ (face), matrix of $512 \times 512$, and a field of view of $25.0 \mathrm{~mm}$. The axial and coronal images showed irregular and hyperdense growth of the right hemiface with involvement of the following bones: zygomatic and frontal bone, temporal and maxillary process, sphenoid, occipital, and maxilla (cheek and palate enlargement). The right temporomandibular condyle and coronoid process were enlarged as well as the mandibular ramus. The coronal image showed hypoplasia of the right maxillary antrum associated with increased vertical dimensions of the orbit (Fig. 2E). In addition, enlargement of the inferior turbinate with nasal septal deviation was observed. Brain CT showed no abnormalities other than enlargement of the frontal and parietal bones (Figs. 2F and G). These extensive clinical and radiographic abnormalities were considered to justify a diagnosis of true HFH.

The patient underwent dental treatment, including restorations and prophylaxis. Initially, gingival plasty was performed for posterior placement of orthodontic and orthopedic appliances to reduce the crossbite. No therapy was instituted for facial deformity, but periodic clinical and radiographic assessment was recommended to the patient. Further orthodontic treatment and surgery will be assessed when the patient stops growing.

\section{Discussion}

HFH may be associated with deformities of the skeletal system, including macrodactyly, polydactyly, syndactyly, scoliosis, tilting of the pelvis, and clubfoot (1). Central nervous system defects may include cerebral enlargement, epilepsy, strabismus, and mental retardation in 15-20\% of patients, and in some cases dilatation of the pupils on the affected side (5). Genitourinary system disorders, such as hypospadias, cryptorchidism, and medullary sponge kidney, are also noted occasionally (6). Our patient had the characteristic clinical features of HFH, such as unilateral overgrowth of the orofacial soft tissues and bones and tongue asymmetry.

The etiology of HFH is unknown, but various theories have been put forward to explain it; including endocrine imbalance, neural abnormalities, asymmetrical cell division and deviation of the twinning process, chromosomal abnormalities, alterations of intrauterine development, and vascular or lymphatic abnormalities (7).

Imaging features of $\mathrm{HFH}$ have been reported. Alterations in dental development with earlier dental maturity on the affected side, early eruption, and idiopathic root resorption have been described (8). Panoramic and periapical radiographs of the present patient showed no alterations involving the dental morphology.
HFH is more common in men than in women and more commonly involves the right side of the face. In keeping with this, the present case was a boy, and the right side of the face was affected. These asymmetries are often noted at birth, but in this case they were noted by the parents when the child was 2 years old. The condition is usually accentuated with age, particularly around puberty (1). In our patient, there were no significant changes elsewhere in the body or in the serum chemistry. Therefore, systemic diseases such as Beckwith-Wiedemann syndrome, Proteus syndrome, Schimmelpenning (epidermal nevus) syndrome, neurofibromatosis, and hyperpituitarism were excluded. There may be overlap of clinical manifestations between HFH and these conditions (2). In Proteus syndrome, the patient has asymmetry of the limbs, overgrowth of the hands or feet or both, lipomas, connective tissue nevi, and vascular and lymphatic malformations (9). In Beckwith-Wiedemann syndrome, patients usually have increased birth weight, postnatal gigantism, macroglossia, omphalocele, and distinctive ear lobe grooves (9). In Schimmelpenning syndrome (epidermal nevus), patients have epidermal nevi; sebaceous nevi are said to be the hallmark of the syndrome. Other common features of Schimmelpenning syndrome are seizures, developmental delay, hemangiomas, kyphosis/scoliosis, and extension of nevus to the eyelid (9).

Congenital HFH is expressed as facial asymmetry resulting from unilateral overgrowth. Yoshimoto et al. (8) compared the proliferative activity of primary cultured osteoblasts taken from the hypertrophic side with those taken from the normal side. They reported that both fetal calf serum and basic fibroblast growth factor stimulated osteoblast DNA synthesis and that cultured osteoblast proliferation was markedly increased on the hypertrophic side. They concluded that fibroblast growth factor and its receptor signal transduction axis may be selectively involved in affected osteoblasts, leading to hypertrophy.

Procedures are usually planned for when physiologic growth ceases. These may include reconstructive procedures such as ostectomy or orthognathic surgical procedures. Other options including facelifts and orthognathic surgical procedures in conjunction with orthodontic therapy have been reported in the literature (10). Cosmetic reconstruction has frequently been performed with limited to moderate gains (1). Our patient received medical and dental care without surgical intervention.

$\mathrm{HFH}$ is generally associated with a good prognosis (1); an extensive search of the English language literature revealed no formal reports of malignant degeneration. The asymmetry usually remains constant up to the end of adolescence and the condition stabilizes thereafter (1).

In summary, we report a case of HFH in an 8-year-old 
white boy with an abnormal shape of the jaw and face on the right side. The patient showed no other systemic or syndromic alterations. The clinical features were similar to those described in the scientific literature. However, the patient had no dental abnormalities of size or morphology. Affected individuals generally require lifelong management by a multidisciplinary team of health care specialists.

\section{Acknowledgments}

This work was supported in part by grants from the State Minas Gerais Research Foundation (FAPEMIG), Belo Horizonte, Brazil and CNPq.

\section{References}

1. Pollock RA, Newman MH, Burdi AR, Condit DP (1985) Congenital hemifacial hyperplasia: an embryologic hypothesis and case report. Cleft Palate J 22, 173-184.

2. Islam MN, Bhattacharyya I, Ojha J, Bober K, Cohen DM, Green JG (2007) Comparison between true and partial hemifacial hypertrophy. Oral Surg Oral Med Oral Pathol Oral Radiol Endod 104, 501-509.

3. Rowe NH (1962) Hemifacial hypertrophy. Review of the literature and addition of four cases. Oral Surg Oral Med Oral Pathol 15, 572-587.

4. Bergman JA (1973) Primary hemifacial hypertrophy.
Review and report of a case. Arch Otolaryngol 97, 490-494.

5. Hayashi S, Tomioka T, Aoki H, Nakakuki K, Mekaru K (1973) Hemifacial hypertrophy. Report of two cases. Oral Surg Oral Med Oral Pathol 35, 750-761.

6. Eisenberg RL, Pfister RC (1972) Medullary sponge kidney associated with congenital hemihypertrophy (asymmetry). A case report and survey of the literature. Am J Roentgenol Radium Ther Nucl Med 116, 773-777.

7. Lee S, Sze R, Murakami C, Gruss J, Cunningham M (2001) Hemifacial myohyperplasia: description of a new syndrome. Am J Med Genet 103, 326-333.

8. Yoshimoto H, Yano H, Kobayashi K, Hirano A, Motomura K, Ohtsuru A, Yamashita S, Fujii T (1998) Increased proliferative activity of osteoblasts in congenital hemifacial hypertrophy. Plast Reconstr Surg 102, 1605-1610.

9. Siponen M, Sándor GKB, Ylikontiola L, Salo T, Tuominen H (2007) Multiple orofacial intraneural perineuriomas in a patient with hemifacial hyperplasia. Oral Surg Oral Med Oral Pathol Oral Radiol Endod 104, e38-44.

10. Hall HD (1984) An improved method for treatment of facial asymmetry secondary to jaw deformity. $\mathrm{J}$ Oral Maxillofac Surg 42, 673-679. 